\title{
Experiments with Adaptable Interfaces for Elderly People*
}

\author{
Norbert Kuhn, Stefan Richter, Michael Schmidt, Andreas Truar, \\ Markus Schwinn, Stefan Naumann, and Markus Dick \\ Institut für Softwaresysteme in Wirtschaft, Umwelt und Verwaltung \\ Fachhochschule Trier, Umwelt-Campus Birkenfeld, \\ Campusallee, 55761 Birkenfeld \\ \{n.kuhn, s.richter,m.schmidt, a.truar,m.schwinn, s.naumann,m.dick\} \\ aumwelt-campus.de
}

\begin{abstract}
The demographic change in European societies has led to raise attention for the needs of elderly people. Many of elderly people suffer from cognitive as well as from physical restrictions. This impacts their ability to participate and to communicate in their social environment. This could be substituted by constructing a virtual social environment based on services like videoconferences, chat rooms, e-mail and so on. However, elderly people often have difficulties to use IT technology because they encounter restrictions caused by their age. Thus, they need specific and adaptable human computer interfaces to be able to interact with IT systems. We present here some ideas how such interfaces can be constructed with reasonable effort and discuss some results from an evaluation of these ideas for an implementation of an interface to a personal information system for elderly and handicapped users.
\end{abstract}

Keywords: Adaptable HCI systems, Multi-modal User Interfaces, Accessibility, Ambient Assisted Living.

\section{Introduction}

Since many years a dramatic change in the age structure of the European nations may be observed. [1]. A further effect of the ageing society is the increasing percentage of people with disabilities in vision and hearing. [2].

In contrast to the generation of the "digital natives" elderly users often have rare experience e.g. in communication in social networks, or electronic communication at all. This means that classical criteria for human computer interfaces, like usability, clarity of the navigation structure etc are crucial for the acceptance of any application for elderly people.

Furthermore, we state that also techniques to ensure accessibility to IT systems are important to be considered. In [3][4] we presented some ideas to provide visual impaired users with access to printed documents.

* This work is supported by the German Ministry of Research and Technology under grant FKZ $1771 \mathrm{X} 07$. 
In this paper however, we focus on the construction of the user interface. This should be able to embed an application into the experienced context of a particular user and allow him to use a wide rage of devices to interact with an application.

\section{Constructing Dynamic Interfaces}

\subsection{Automatic Adaption of User Interfaces}

Based on our evaluation with elderly people the process of automatic adaption of the user interface has to consider two main aspects. The first aspect concerns the presentation of the user interface, while the second aspect concerns the integration of different input devices. For the automatic adaption of the presentation we use a model-based approach. For the integration of different input devices we use the idea of abstract events.

\subsection{Model-Based Design}

In a model-based approach the user interface is usually described by a set of different models. For example, in [7], Calvary et. al. make use of four models, which are arranged in a hierarchical order. Each model describes the user interface on a different level of abstraction.

With this approach it is possible to adapt the user interface to different output devices, e.g. a PDA, a PC or a TV. In our context we want to specialize this approach to consider the input devices and the user's needs. Therefore, we introduce the concepts of a user model and a hardware model.

\subsection{User/Hardware Model}

The user model describes the requirements of a particular user concerning the user interface. For the group of elderly people we mentioned already the heterogeneity of requirements cased by a smooth but continuous degrade of their physical, perceptual, and cognitive abilities.

The hardware model contains information about the users hardware configuration. Like the user model the hardware model is different for every user. This is caused be the different input/output devices that are used by the intended user group.

For the realization of an automatically adaption of the user interface a model-based approach which considers the user model and the hardware model should be used.

\subsection{Enhanced User Model for Seniors}

To realize the dynamic approach, a model of the user interface is needed. Our framework extend the work of Calvary et. al.[8]

At the top of this hierarchy there is the Interaction Model. It describes the interactions between the user and the user interface in an abstract way. In addition to the reference framework of Calvary et. al. the Interaction Model is expanded with a user model. This model describes all the abilities and needs for a concrete user. 


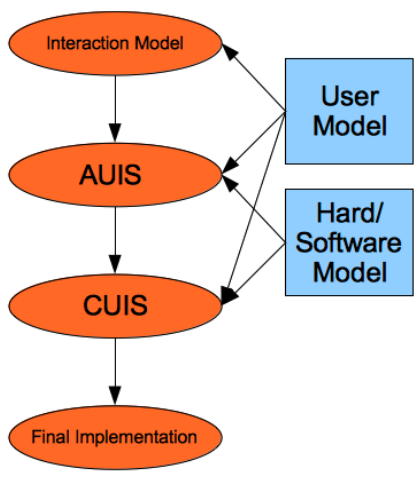

Fig. 1. Reference framework for the model based approach

The next level of hierarchy is the AUIS (Abstract User Interface for seniors). In this level the interactions of the Interaction Model are specified using abstract interface objects. The model consists of basic interactions that occur inside a user interface. As shown in Fig. 1 the user model and the hardware model are used while building the AUIS.

In the next stage of the framework there is the CUIS (Concrete User Interface for seniors). At this level the abstract user interface objects are mapped to concrete user interface objects. Like the two models before the CUIS also includes the user model and the hardware model. This is important because a specific user may change the mapping between the AUIS and the CUIS.

The last stage addresses the final implementation. Here, the CUIS is transformed to a final implementation, which runs on a specific hardware configuration.

In conjunction with the user model and the hardware model the framework will be able to automatically adapt the views of a user interface.

\subsection{Adaption to Different Input Devices Using Abstract Events}

Most applications are designed to use a mouse and/or a keyboard. In many cases it is very difficult or even impossible to use alternative input devices, such as a graphic tablet, a microphone or a remote control for basic functions. To overcome this limitation we introduce the idea of an abstract event. The abstract events are the same for every new device presented to the system. In this way the user interface has to support only these abstract events. To realize this, the special events of an input device are mapped to abstract events.

To implement this mapping we may use the Observer Design Pattern as described in the GOF Patterns ([5][6]).

This software architecture allows the user to use all input devices simultaneously. Furthermore, a new device can be easily integrated through a concrete implementation of the specified events.

The framework described here makes it possible to build the user interface at runtime. This ensures that the user interface can always adapt to the user's needs. 


\section{Conclusion and Future Work}

The feedback of elderly or handicapped users to our demonstrators has underlined the need of adaptable interfaces. The approach we discussed here uses a model-based design and considers the user's needs, and the type of device he uses. It allows the implementation of an adaptable, dynamically generated user interface with special focus on elderly people.

To proof this concept we will extend our demonstrators to an information serving and document archiving application.

\section{References}

[1] U.S. Census Bureau: International Database, http: / /www. census.gov/ipc/www/idb/pyramids.html

[2] Schweizerische Eidgenossenschaft, Bundesamt für Statistik: State of Health - Severe disabilities in the population older than 15 years, state (2007) (in German), http: / /www.bfs.admin.ch/bfs/portal/de/index/themen/14/02/ $01 / \mathrm{key} / 04 . \mathrm{html}$

[3] Kuhn, N., Richter, S., Schmidt, M., Truar, A.: Improving Accessibility to Governmental Forms. In: IFIP 20th World Computer Congress, Proceedings of the 1st TC 13 HumanComputer Interaction Symposium (HCIS 2008), Milano, Italy, pp. 119-128 (2008), ISBN: 978-0-387-09677-3

[4] Richter, S., Kuhn, N., Naumann, S., Schmidt, M., Truar, A.: Enhancing Accessibility to EGovernment Processes. International Journal of Informations Communication Technologies and Human Development, Special Issue: ICT and E-Governance 1(2), 28-47 (2009)

[5] Gamma, E., Helm, R., Johnson, R., Vlissides, J.: Design Patterns Elements of Reusable Object-Oriented Software, pp. 293-303. Addison-Wesley, Reading (2007) (35th Printing)

[6] Kuchana, P.: Software Architecture Design Patterns in Java, pp. 343-354. CRC Press LLC, Boca Raton (2004)

[7] Collignon, B., Vanderdonckt, J., Calvary, G.: Model-Driven Engineering of Multi-Target Plastic User Interfaces. In: Fourth International Conference on Autonomic and Autonomous Systems, ICAS (2008)

[8] Calvary, G., Coutaz, J., Thevenin, D., Limbourg, Q., Bouillon, L., Vanderdonckt, J.: A unifying reference framework for multi-target user interfaces. Interacting with Computers 15, 289-308 (June) 